https://helda.helsinki.fi

\title{
Chaotic attractors in the four-dimensional Leslie-Gower competition model
}

\section{Gyllenberg, Mats}

2020-01-15

Gyllenberg , M , Jiang , J \& Niu , L 2020 , ' Chaotic attractors in the four-dimensional Leslie-Gower competition model ' , Physica D: Nonlinear Phenomena , vol. 402 , 132186 . https://doi.org/10.1016/j.p

http://hdl.handle.net/10138/312748

https://doi.org/10.1016/j.physd.2019.132186

acceptedVersion

Downloaded from Helda, University of Helsinki institutional repository.

This is an electronic reprint of the original article.

This reprint may differ from the original in pagination and typographic detail.

Please cite the original version. 


\title{
Chaotic attractors in the four-dimensional Leslie-Gower competition model
}

\author{
Mats Gyllenberg ${ }^{\mathrm{a}}$, Jifa Jiang ${ }^{\mathrm{b}}$, Lei Niu ${ }^{\mathrm{a}, *}$ \\ ${ }^{a}$ Department of Mathematics and Statistics, University of Helsinki, Helsinki FI-00014, Finland \\ ${ }^{b}$ Mathematics and Science College, Shanghai Normal University, Shanghai 200234, China
}

\begin{abstract}
We study the occurrence of the chaotic attractor in the four-dimensional classical LeslieGower competition model. We find that chaos can be generated by a cascade of quasiperioddoubling bifurcations starting from a supercritical Neimark-Sacker bifurcation of the positive fixed point in this model. The chaotic attractor is contained in the three-dimensional carrying simplex, that is a globally attracting invariant manifold. Biologically, the result implies that the invasion attempts by an invader into a trimorphic population under the Leslie-Gower dynamics can lead to chaos.
\end{abstract}

Keywords: Leslie-Gower model, carrying simplex, chaotic attractor, quasiperiod-doubling cascades, invasion

2010 MSC: 92D25, 37D45, 37Gxx

\section{Introduction}

2 Consider the discrete-time dynamical system modelling the interaction of $n$ species 3 induced by the map of Kolmogorov type

$$
T(x)=\left(x_{1} F_{1}(x), \ldots, x_{n} F_{n}(x)\right), \quad x \in \mathbb{R}_{+}^{n}:=[0, \infty)^{n},
$$

${ }_{4}$ where $F_{i}$ are continuous with $F_{i}(x)>0$ for all $x \in \mathbb{R}_{+}^{n}, i=1, \ldots, n$. Here, $x$ denotes

5 the vector of populations at one generation, and $T(x)$ is the corresponding vector at the

6 next generation. The map $T$ is called a competitive map in a subset $W \subset \mathbb{R}_{+}^{n}$ if for

7 all $x, y \in W$ such that $T_{i}(x) \leq T_{i}(y)$ for all $i=1, \ldots, n$ but $T(x) \neq T(y)$ one has that

\footnotetext{
* Corresponding author

Email address: lei.niu@helsinki.fi (Lei Niu)
} 
s $x_{i}<y_{i}$ provided $y_{i}>0$. Since the early work of Hirsch [1] and Smith [2], it is well

9 known that most competitive maps admit an invariant hypersurface $\Sigma$ of codimension 10 one, known as the carrying simplex, which attracts all nontrivial orbits (see, for instance, 11 [3, 4, 5, 6, 7, 8, 9, ). The origin 0 is a repeller for $T$, and $\Sigma$ is the boundary in $\mathbb{R}_{+}^{n}$ of the 12 basin of repulsion of the origin which satisfies the following properties:

$13 \quad(\mathrm{P} 1) \Sigma$ is compact and unordered, i.e. if $x, y \in \Sigma$ such that $x-y \in \mathbb{R}_{+}^{n}$, then $x=y$;

14 (P2) $\Sigma$ is homeomorphic via radial projection to the $(n-1)$-dimensional standard probability simplex $\Delta^{n-1}=\left\{x \in \mathbb{R}_{+}^{n}: \sum_{i=1}^{n} x_{i}=1\right\} ;$

$16 \quad$ (P3) $\forall x \in \mathbb{R}_{+}^{n} \backslash\{0\}$, there exists some $y \in \Sigma$ such that $\lim _{k \rightarrow \infty}\left|T^{k}(x)-T^{k}(y)\right|=0$;

17 (P4) $T(\Sigma)=\Sigma$, and $T: \Sigma \mapsto \Sigma$ is a homeomorphism.

18 In particular, the classical discrete-time models admitting a carrying simplex include the

19 Leslie-Gower model (see [9])

$$
T_{i}(x)=\frac{\left(1+r_{i}\right) x_{i}}{1+\sum_{j=1}^{n} b_{i j} x_{j}}, r_{i}, b_{i j}>0, i, j=1, \cdots, n,
$$

20 the Atkinson-Allen model (see [10])

$$
T_{i}(x)=\frac{\left(1+r_{i}\right)\left(1-a_{i}\right) x_{i}}{1+\sum_{j=1}^{n} b_{i j} x_{j}}+a_{i} x_{i}, 0<a_{i}<1, r_{i}, b_{i j}>0, i, j=1, \cdots, n,
$$

21 and the Ricker model (under mild conditions) (see [5, 6, 8,

$$
T_{i}(x)=x_{i} \exp \left(r_{i}\left(1-\sum_{j=1}^{n} b_{i j} x_{j}\right)\right), r_{i}, b_{i j}>0, i, j=1, \cdots, n .
$$

The importance of the existence of a carrying simplex stems from the fact that it contains all the interesting dynamics, such as the periodic orbits, quasiperiodic orbits and heteroclinic cycles. For 1D (i.e. $n=1$ ) competitive maps admitting a carrying simplex, there is a globally attracting positive fixed point as the carrying simplex, so the dynamics is trivial. For $2 \mathrm{D}$ competitive maps admitting a carrying simplex, every trajectory also converges to a fixed point (see [11), because the map restricted to the one-dimensional carrying simplex is a homeomorphism. For 3D competitive maps admitting a carrying simplex, nontrivial dynamics such as Neimark-Sacker bifurcations and heteroclinic cycles can occur (see, for example, $8,9,10,11,12$ ). Neimark-Sacker bifurcation is the birth 
Lotka-Volterra competition model

$$
\frac{d x_{i}(t)}{d t}=x_{i}(t)\left(\beta_{i}-\sum_{j=1}^{n} \alpha_{i j} x_{j}(t)\right), \beta_{i}, \alpha_{i j}>0, i, j=1, \ldots, n .
$$

${ }_{41}$ In [9], Jiang and Niu studied the 3D Leslie-Gower model (2), which was classified into ${ }_{42} 33$ stable equivalence classes in terms of inequalities on the parameters $r_{i}$ and $b_{i j}$ via 43 the boundary dynamics on the $2 \mathrm{D}$ carrying simplex. In classes $26-31$, Neimark-Sacker 44 bifurcations can occur, while in classes 1-25 and 33, every nontrivial trajectory converges 45 to a fixed point on the carrying simplex. Their results show that the 3D Leslie-Gower 46 model (2) also has the similar dynamic scenarios as the 3D Lotka-Volterra competition 47 model (5) (see [18, 19]). The reader can consult, for instance, [20, 21, 22, 23, 24, 25, 26.

48 27, for more results on the Lotka-Volterra competition model (5).

It is known that chaos cannot occur in the 3D competitive Lotka-Volterra model 50 (5) by Hirsch's carrying simplex theory for competitive continuous flow in [1] and the 51 Poincaré-Bendixson theorem for planar continuous flow. However, though the Leslie52 Gower model (2) has a carrying simplex, it can not guarantee that there is no chaos 53 in the 3D Leslie-Gower model (2) since the Poincaré-Bendixson theorem does not hold 54 for discrete-time systems. The results in [9, 13] seem to imply that the chaos may not 55 occur in the 3D Leslie-Gower model (2). On the other hand, we have carried out a

of an invariant cycle from a fixed point in discrete-time systems, and either all orbits are pense on the invariant cycle (in this case, it is called a can occur, that is every orbit converges to a fixed point, when there is no positive fixed point or there is a unique positive fixed point whose index is -1 by the topological results on homeomorphisms of the plane. Criteria on the global stability of the positive fixed oint are provided in [14] and [15].

The 2D Leslie-Gower model [2] was studied by Liu and Elaydi [16] and Cushing et al. [17] in detail, which has the same dynamic scenarios as the $(2 \mathrm{D}$, i.e. $n=2)$ Lotka-Volterra competition model 
carried out lots of numerical simulations for the Poincaré map of the three-dimensional

${ }_{61}$ periodic Lotka-Volterra competition model [5], that is the positive parameters $\beta_{i}, \alpha_{i j}$ in

62 (5) are time-periodic with the same period, which also admits a carrying simplex by 3,5 .

63 Periodic orbits and quasiperiodic curves, corresponding to subharmonic and quasiperiodic

${ }_{64}$ solutions of the periodic Lotka-Volterra model, were detected for the Poincaré map, but

${ }_{65}$ the chaotic attractor has not been found. It seems to us that there might be no chaos

${ }_{66}$ in these classical 3D competitive discrete-time systems admitting a carrying simplex

${ }_{67}$ by our numerical experiments and the results in [9, 10, 13, 18, comparing with the 3D

${ }_{68}$ competitive continuous-time systems (see $[18,19,28$ for example). Therefore, we turn to

69 explore the occurrence of chaos in the 4D classical discrete-time models, and we find that

70 the chaotic attractor can occur in the Leslie-Gower model (2) of four mutually competing

71 species.

${ }_{72}$ Specifically, consider the 4D Leslie-Gower competition model $T$ given by

$$
T_{i}(x)=\frac{\left(1+r_{i}\right) x_{i}}{1+\sum_{j=1}^{4} r_{i} a_{i j} x_{j}}, r_{i}, a_{i j}>0, i, j=1,2,3,4
$$

73 with

$$
r_{i}=[0.08, \mu, 0.15,0.28],
$$

74 and

$$
a_{i j}=\left[\begin{array}{cccc}
2.58 & 4.25 & 3.72 & 0.085 \\
7.3 & 2.75 & 0.01 & 1.82 \\
0.074 & 1.19 & 3.32 & 4.64 \\
3.8 & 1.08 & 1.7 & 3.64
\end{array}\right]
$$

75 where $\mu>0$ is a free parameter. We find that chaos can occur in this Leslie-Gower model

76 of four competing species for some $\mu$. For example, when $\mu=0.36$, a chaotic attractor

77 is detected which is shown in Fig. 1 . The corresponding Lyapunov exponents which can

78 be computed by using MatContM [29, 30] based on the QR method (see [31, 32]) are

79 0.0011, 0, -0.0463 and -0.1751 , which implies a Lyapunov dimension (see [31, 33]) of

80 2.024. This means that the invertible bounded 4D Leslie-Gower map of type (2) can has

81 a $3 \mathrm{D}$ carrying simplex containing a chaotic attractor. 


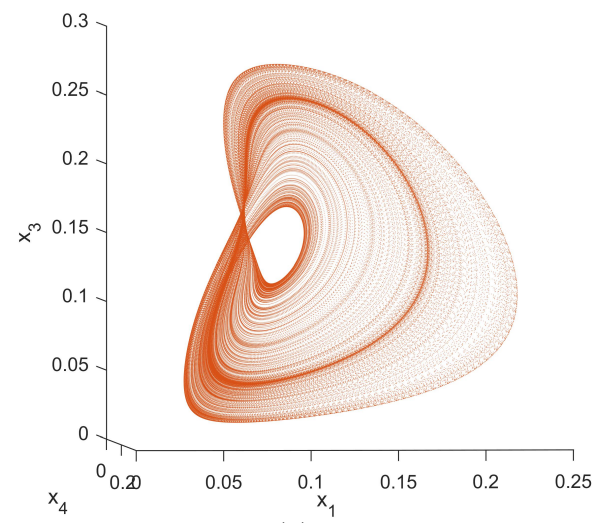

(a)

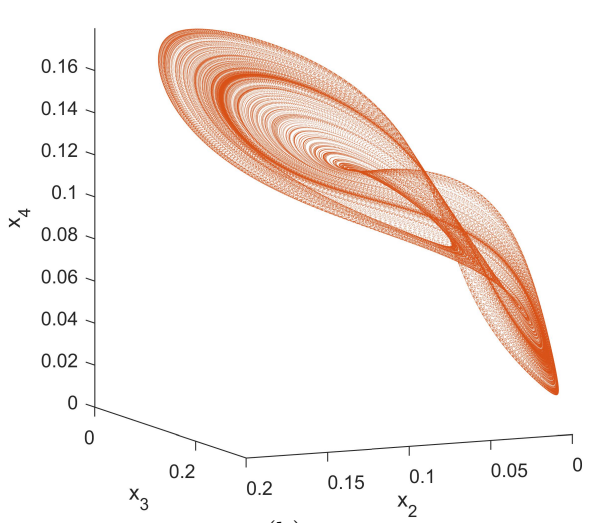

(b)

Figure 1: A chaotic attractor occurs at $\mu=0.36$. (a) The projection of the chaotic attractor on the $x_{1} x_{3} x_{4}$ space. (b) The projection of the chaotic attractor on the $x_{2} x_{3} x_{4}$ space.

\section{Derivation of the Leslie-Gower model}

In this section, we give a mechanistic derivation of the Leslie-Gower model induced 84 by the map (2) from first principles.

${ }_{85}$ We assume that the season is divided into two periods: one of competition and one ${ }_{86}$ of reproduction. During the first period, the individuals of $n$ species do not die, but 87 compete for $n$ different resources $R_{j}, j=1, \ldots, n$. Assuming chemostat dynamics of the ${ }_{88}$ resources and a Holling type I functional response, we obtain the following system of 89 ordinary differential equations for the resource dynamics:

$$
\frac{d R_{i}}{d t}=D_{i}\left(\bar{R}_{i}-R_{i}\right)-R_{i} \sum_{j=1}^{n} a_{i j} x_{j}, \quad i=1, \ldots, n .
$$

90 Assuming the resource dynamics is fast, the resource concentrations will have reached 91 the following steady state by the end of the period of competition:

$$
R_{i}=\frac{\bar{R}_{i}}{1+\sum_{j=1}^{n} \frac{a_{i j}}{D_{i}} x_{j}}, \quad i=1, \ldots, n .
$$

92 During the period of reproduction, individuals of species $i$ will use only the resource $R_{i}$ 93 given by (10) to produce offspring (with a conversion factor $\gamma_{i}$ ) born at the beginning of

94 the next period of competition and then die. Putting all these assumptions together, we

${ }_{95}$ finally arrive at the following map $T$ taking the state of the community at the beginning 
of one period of competition to the next one:

$$
T_{i}(x)=\frac{\gamma_{i} \bar{R}_{i} x_{i}}{1+\sum_{j=1}^{n} \frac{a_{i j}}{D_{i}} x_{j}}, i=1, \ldots, n,
$$

97

which is, of course, exactly the map 22, but with the parameters denoted in a different way. In this paper we mainly discuss the occurrence of the chaos and the route to chaos in the Leslie-Gower model of four competing species, so we shall keep the parameters of (6) to make comparison with the similar models treated in the papers mentioned above easier.

\section{Preliminaries}

Let $\dot{\mathbb{R}}_{+}^{n}=\left\{x \in \mathbb{R}_{+}^{n}: x_{i}>0, i=1, \ldots, n\right\}$ be the open positive cone and $\pi_{i}=\{x \in$ $\left.\mathbb{R}_{+}^{n}: x_{i}=0\right\}$ be the $i$ th coordinate plane. Let $\dot{\Sigma}=\Sigma \cap \dot{\mathbb{R}}_{+}^{n}$ denote the interior of the carrying simplex $\Sigma$ and $\partial \Sigma=\Sigma \backslash \dot{\Sigma}$ denote the boundary of $\Sigma$.

Lemma 1 (see [9, Corollary 3.1]). Any n-dimensional Leslie-Gower model (2) has a $(n-1)$-dimensional carrying simplex $\Sigma$ satisfying the properties $(\mathrm{P} 1)-(\mathrm{P} 4)$ in section 1 .

Lemma 1 implies that the long-term behavior of the Leslie-Gower model (2) is determined by the dynamics on $\Sigma$, and the nonzero forward limit sets in $\mathbb{R}_{+}^{n}$ all lie on $\Sigma$. In particular, for the $4 \mathrm{D}$ Leslie-Gower model $T$ given by (6), it has a $3 \mathrm{D}$ carrying simplex $\Sigma$ which is homeomorphic to the tetrahedron $\Delta^{3}$ (see Fig. (4)). Note that each coordinate plane $\pi_{i}$ is positively invariant under $T$, and $\left.T\right|_{\pi_{i}}$ is a $3 \mathrm{D}$ Leslie-Gower model which has a $2 \mathrm{D}$ carrying simplex homeomorphic to the triangle $\Delta^{2}$, where $\left.T\right|_{\pi_{i}}$ is the restriction of $T$ to $\pi_{i}$. Moreover, the boundary of $\Sigma$ for $T$ is composed of the $2 \mathrm{D}$ carrying simplices of $\left.T\right|_{\pi_{i}}$, i.e. $\partial \Sigma=\cup_{i=1}^{4}(\partial \Sigma)_{i}$, where $(\partial \Sigma)_{i}=\partial \Sigma \cap \pi_{i}$ is the carrying simplex of $\left.T\right|_{\pi_{i}}$. It means that the dynamics on the boundary of $\Sigma$ is determined by the four 3D Leslie-Gower maps $\left.T\right|_{\pi_{i}}, i=1,2,3,4$.

Consider the general 3D Leslie-Gower model $\hat{T}$ on $\mathbb{R}_{+}^{3}$ given by

$$
\hat{T}_{i}(x)=\frac{\left(1+r_{i}\right) x_{i}}{1+\sum_{j=1}^{3} r_{i} a_{i j} x_{j}}, r_{i}, a_{i j}>0, i, j=1,2,3 .
$$


Let $\hat{\Sigma}$ be the carrying simplex of $\hat{T}$. It is easy to see that there are three axial fixed points $v_{\{1\}}, v_{\{2\}}, v_{\{3\}}$ with $v_{\{i\}}$ lying on the positive $x_{i}$-axis, i.e.,

$$
v_{\{1\}}=\left(\frac{1}{a_{11}}, 0,0\right), v_{\{2\}}=\left(0, \frac{1}{a_{22}}, 0\right), \text { and } v_{\{3\}}=\left(0,0, \frac{1}{a_{33}}\right),
$$

which are the vertices of the carrying simplex $\hat{\Sigma}$. There may exist a planar fixed point $s_{\{j k\}}$ lying on the positive cone of the $x_{j} x_{k}$ subspace, i.e. the interior of $\pi_{i}$, and there may also exist a positive fixed point $p$ in $\dot{\mathbb{R}}_{+}^{3}$.

Set

$$
\gamma_{i j}=a_{i i}-a_{j i}, \quad \beta_{i j}=\frac{a_{j j}-a_{i j}}{a_{i i} a_{j j}-a_{i j} a_{j i}} .
$$

Lemmas 2 and 3 below follow from [9, Lemmas 4.5-4.7] immediately.

Lemma 2. If $\gamma_{i j}>0($ resp. $<0)$ then $v_{\{i\}}$ repels (resp. attracts) along $\partial \Sigma \cap \pi_{k}$, and moreover, $v_{\{i\}}$ is hyperbolic if and only if $\gamma_{i j} \gamma_{i k} \neq 0$, where $i, j, k$ are distinct.

Lemma 3. If $\gamma_{j k} \gamma_{k j}>0$ then $\hat{T}$ has a planar fixed point $s_{\{j k\}}$ in the interior of $\pi_{i}$, where $i, j, k$ are distinct. Moreover, if $\gamma_{j k}, \gamma_{k j}<0$ (resp. $\left.>0\right)$ then $s_{\{j k\}}$ repels (resp. attracts) along $\partial \Sigma$; if $a_{i j} \beta_{j k}+a_{i k} \beta_{k j}<1$ (resp. $>1$ ) then $s_{\{j k\}}$ locally repels (resp. attracts) in $\dot{\Sigma} ; s_{\{j k\}}$ is hyperbolic if and only if $a_{i j} \beta_{j k}+a_{i k} \beta_{k j} \neq 1$.

Remark 3.1. The biological meaning of the condition $\gamma_{i j}>0($ resp. $<0)$ is that species $j$ can (resp. cannot) invade the monomorphic population of species $i$ at the steady state $v_{\{i\}}$; here $i, j$ are distinct. Suppose $s_{\{j k\}}$ exists which is a stable coexistence of species $j$ and $k$ in the absence of species $i$ (for example, if $\gamma_{j k}, \gamma_{k j}>0$ then $s_{\{j k\}}$ exists and it is stable in the interior of $\left.\pi_{i}\right)$. In this case, the biological meaning of the condition $a_{i j} \beta_{j k}+$ $a_{i k} \beta_{k j}<1$ (resp. $>1$ ) is that the species $i$ can (resp. cannot) invade the dimorphic population set by species $j$ and $k$ at the steady state $s_{\{j k\}}$; here $i, j, k$ are distinct. A mathematical framework for treating questions of invasion and evolution that explicitly takes the population dynamics into account is adaptive dynamics; see [34, 35, 36, 37, for more details.

Let $S, \hat{S}$ be two 3D Leslie-Gower maps of type 12. $S$ and $\hat{S}$ are said to be equivalent relative to $\partial \hat{\Sigma}$ if there exists a permutation $\sigma$ of $\{1,2,3\}$ such that $S$ has an axial fixed point $v_{\{i\}}$ (resp. a planar fixed point $s_{\{j k\}}$ ) if and only if $\hat{S}$ has an axial fixed point 
142

$\hat{v}_{\{\sigma(i)\}}$ (resp. a planar fixed point $\hat{s}_{\{\sigma(j) \sigma(k)\}}$ ), and furthermore, $v_{\{i\}}$ (resp. $s_{\{j k\}}$ ) has the same hyperbolicity and local dynamics as $\hat{v}_{\{\sigma(i)\}}$ (resp. $\hat{s}_{\{\sigma(j) \sigma(k)\}}$ ). The map $S$ is said to be stable relative to $\partial \hat{\Sigma}$ if all the fixed points on $\partial \hat{\Sigma}$ are hyperbolic. We say that an equivalence class is stable if each mapping in it is stable relative to $\partial \hat{\Sigma}$.
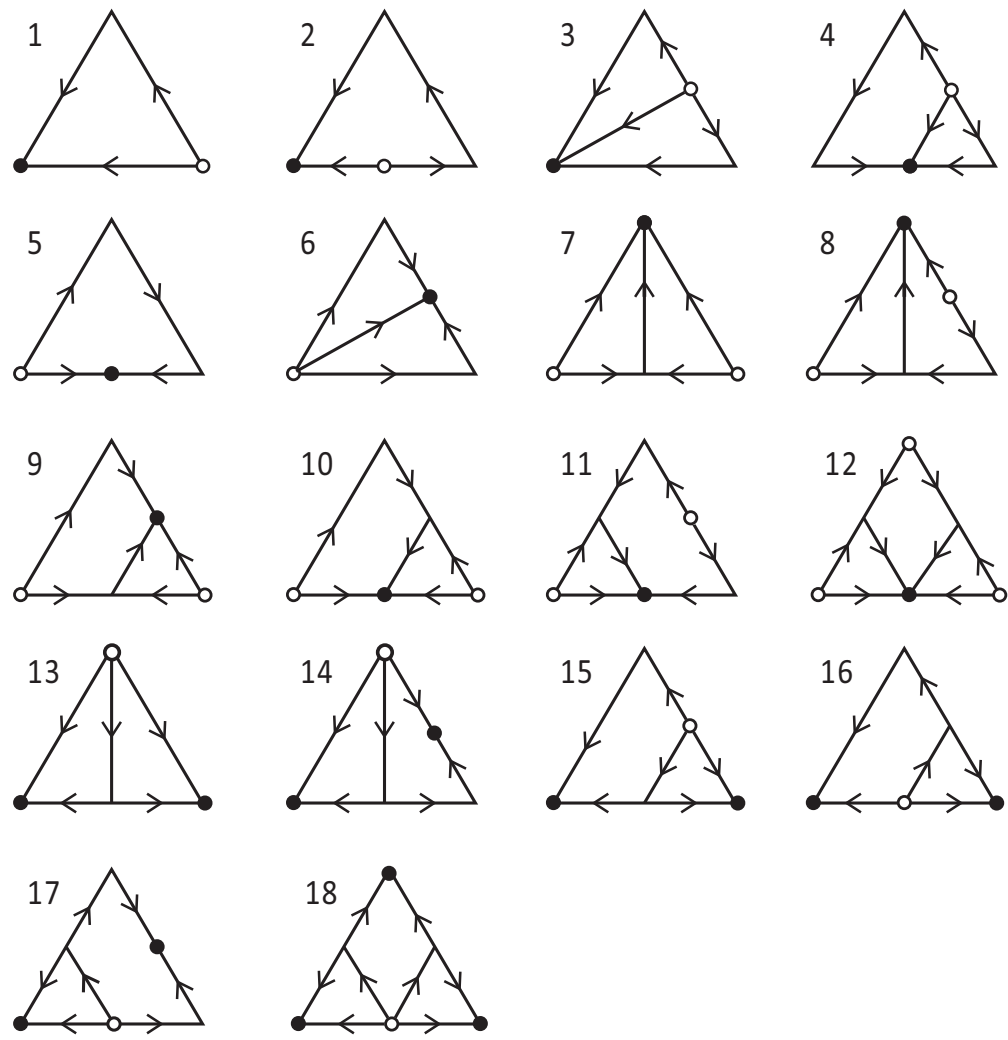

Figure 2: Carrying simplex dynamics in the equivalence classes $1-18$ of the 3D Leslie-Gower model 12. with no positive fixed point. Redrawn from [9]. An attracting fixed point is denoted by a closed dot •, a repelling fixed point by an open dot $\circ$, and a saddle fixed point by the intersection of its stable and unstable manifolds.

Fig. 2 and Fig. 3 show the 33 stable equivalence classes via the boundary dynamics provided in 9]. In classes $1-25$ and 33, only trivial dynamics can occur, that is every orbit converges to a fixed point [9, 13, 15, 38. In class 33, there is a stable coexistence of the three species, while in classes $1-25$, at least one species is driven to extinction. Neimark-Sacker bifurcations can occur in classes $26-31$ but cannot occur in class 32 , and in class 32 the positive fixed point is a repeller. In class 27 , there is a heteroclinic 

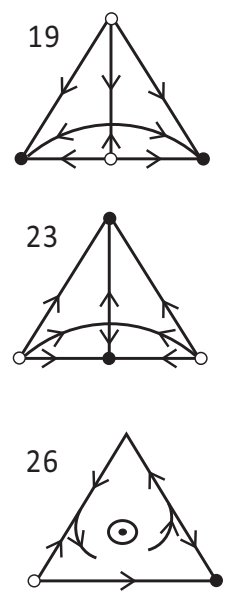

30

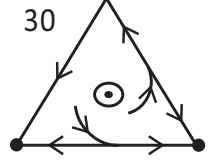

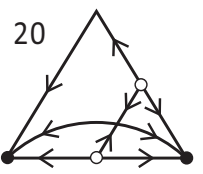
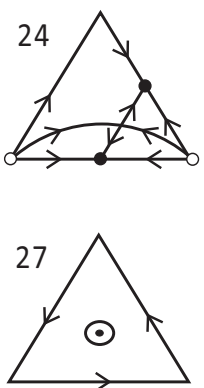

31

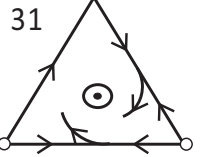

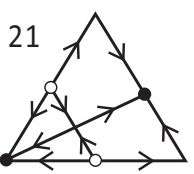
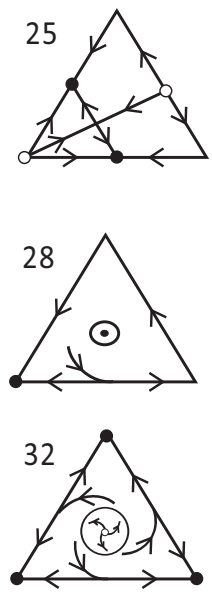

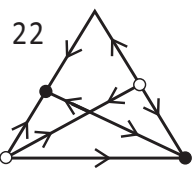

29
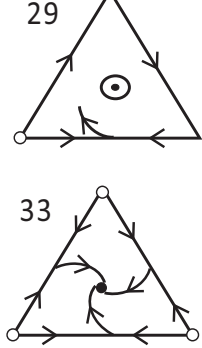

Figure 3: Carrying simplex dynamics in the equivalence classes $19-33$ of the 3D Leslie-Gower model 12. with a positive fixed point. Redrawn from 9, 15 38. The fixed point notation is as in Fig. 2

cycle which is the boundary of the carrying simplex. The symbol $\odot$ in classes $26-31$ represents a region of unknown dynamics. It means that the positive fixed point may be attracting, neutral or repelling on the carrying simplex and in a neighborhood of the positive fixed point, there may be invariant closed curves.

According to 9 and the above arguments, we specifically provide here the dynamics and the parameter conditions for the classes 4,8,9 and 27 of the 3D model $(12)$ which will be used below to study the boundary dynamics of the 4D Leslie-Gower model (6).

Lemma 4. The $3 D$ Leslie-Gower map $\hat{T}$ given by 12 is in the class 4 if there is a permutation $\sigma$ of the indices $\{1,2,3\}$, after which $\hat{T}$ satisfies

(i) $\gamma_{12}>0, \gamma_{13}<0, \gamma_{21}>0, \gamma_{23}<0, \gamma_{31}>0, \gamma_{32}<0$;

(ii) $a_{12} \beta_{23}+a_{13} \beta_{32}<1$;

(iii) $a_{31} \beta_{12}+a_{32} \beta_{21}>1$.

In this case, there are two planar fixed points $s_{\{12\}}, s_{\{23\}}$ and there is no positive fixed point. Moreover, $s_{\{12\}}$ is globally attracting on $\dot{\mathbb{R}}_{+}^{3}$. The phase portrait on $\hat{\Sigma}$ is shown in 
Fig. 2-4, where the vertices can be interpreted as species 1: bottom left, species 2: bottom right, species 3: top.

Lemma 5. The $3 D$ Leslie-Gower map $\hat{T}$ given by $(12)$ is in the class 8 if there is a permutation $\sigma$ of the indices $\{1,2,3\}$, after which $\hat{T}$ satisfies

(i) $\gamma_{12}>0, \gamma_{13}>0, \gamma_{21}>0, \gamma_{23}<0, \gamma_{31}<0, \gamma_{32}<0$;

(ii) $a_{12} \beta_{23}+a_{13} \beta_{32}<1$;

(iii) $a_{31} \beta_{12}+a_{32} \beta_{21}<1$.

In this case, there are two planar fixed points $s_{\{12\}}, s_{\{23\}}$ and there is no positive fixed point. Moreover, $v_{\{3\}}$ is globally attracting on $\dot{\mathbb{R}}_{+}^{3}$. The phase portrait on $\hat{\Sigma}$ is shown in Fig. 2-8, where the vertices can be interpreted as species 1: bottom left, species 2: bottom right, species 3 : top.

Lemma 6. The 3D Leslie-Gower map $\hat{T}$ given by 12 is in the class 9 if there is a permutation $\sigma$ of the indices $\{1,2,3\}$, after which $\hat{T}$ satisfies

(i) $\gamma_{12}>0, \gamma_{13}>0, \gamma_{21}>0, \gamma_{23}>0, \gamma_{31}<0, \gamma_{32}>0$;

(ii) $a_{12} \beta_{23}+a_{13} \beta_{32}>1$;

(iii) $a_{31} \beta_{12}+a_{32} \beta_{21}<1$.

In this case, there are two planar fixed points $s_{\{12\}}, s_{\{23\}}$ and there is no positive fixed point. Moreover, $s_{\{23\}}$ is globally attracting on $\dot{\mathbb{R}}_{+}^{3}$. The phase portrait on $\hat{\Sigma}$ is shown in Fig. 2.9, where the vertices can be interpreted as species 1: bottom left, species 2: bottom right, species 3: top.

Lemma 7. The 3D Leslie-Gower map $\hat{T}$ given by 12 is in the class 27 if there is a permutation $\sigma$ of the indices $\{1,2,3\}$, after which $\hat{T}$ satisfies

$$
\gamma_{12}>0, \gamma_{13}<0, \gamma_{21}<0, \gamma_{23}>0, \gamma_{31}>0, \gamma_{32}<0
$$

In this case, there is a positive fixed point $p$ and $\partial \hat{\Sigma}$ is a heteroclinic cycle. The phase portrait on $\hat{\Sigma}$ is shown in Fig. 3-27, where the vertices can be interpreted as species 1: bottom left, species 2: bottom right, species 3: top. 
Set $\lambda_{i j}=\frac{\left(1+r_{j}\right) a_{i i}}{r_{j} a_{j i}+a_{i i}}$, where $i \neq j$. Let

$$
\vartheta=\ln \lambda_{12} \ln \lambda_{23} \ln \lambda_{31}+\ln \lambda_{21} \ln \lambda_{13} \ln \lambda_{32} .
$$

189

Lemma 8 ([8]; see also [9]). Assume the $3 D$ Leslie-Gower map $\hat{T}$ is in class 27 . If $\vartheta>0$ (resp. $<0)$, then the heteroclinic cycle $\partial \hat{\Sigma}$ of $\hat{T}$ is repelling (resp. attracting).

\section{Dynamics analysis}

In this section, we will study the dynamics on the carrying simplex for the $4 \mathrm{D}$ LeslieGower model $T$ given by (6) with parameters $r_{i}$ and $a_{i j}$ given in (7) and (8) respectively. The fixed points of the $4 \mathrm{D}$ Leslie-Gower model $T$ given by (6) are determined by the linear equations

$$
x_{i}=0 \quad \text { or } \quad \sum_{j=1}^{4} a_{i j} x_{j}=1, \quad i=1,2,3,4,
$$

which depend only on the parameters $a_{i j}$. Therefore, the fixed points of $T$ are independent of the parameter $\mu$ when the parameters $r_{i}$ and $a_{i j}$ are given in (7) and (8). That is to say, the values of the fixed points do not change as $\mu$ varies. It is easy to see that $T$ has nine boundary fixed points:

- the trivial fixed point 0 ;

- four axial fixed points $v_{\{1\}}, v_{\{2\}}, v_{\{3\}}, v_{\{4\}}$ with $v_{\{i\}}$ lying on positive $x_{i}$-axis;

- three planar fixed points $s_{\{12\}}, s_{\{23\}}, s_{\{24\}}$ with $s_{\{i j\}}$ lying on the positive cone of the $x_{i} x_{j}$ subspace;

- a fixed point $q^{\{2\}} \approx(0.1046,0,0.1946,0.0746)^{\tau}$ on the positive cone of the $x_{1} x_{3} x_{4}$ subspace,

and a unique positive fixed point $p$ with

$$
p \approx(0.08734,0.06352,0.1333,0.1024)^{\tau} .
$$

3 admits a 3D carrying simplex $\Sigma$ homeomorphic to $\Delta^{3}$; see Fig. 4 . All the nontrivial boundary fixed points lie on $\partial \Sigma$ and $p$ lies in $\dot{\Sigma}$. It is easy to check that all the nine boundary fixed points are unstable for all $\mu>0$ by their Jacobian matrices. 


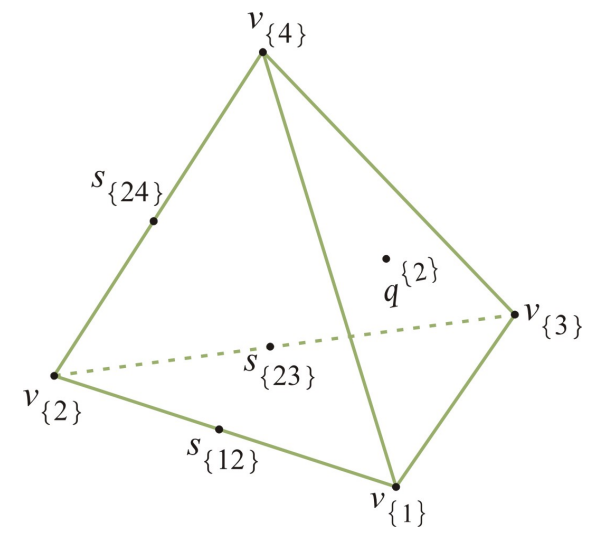

Figure 4: Carrying simplex for the Leslie-Gower map $T$ given by 6. A fixed point on the boundary is represented by a closed dot
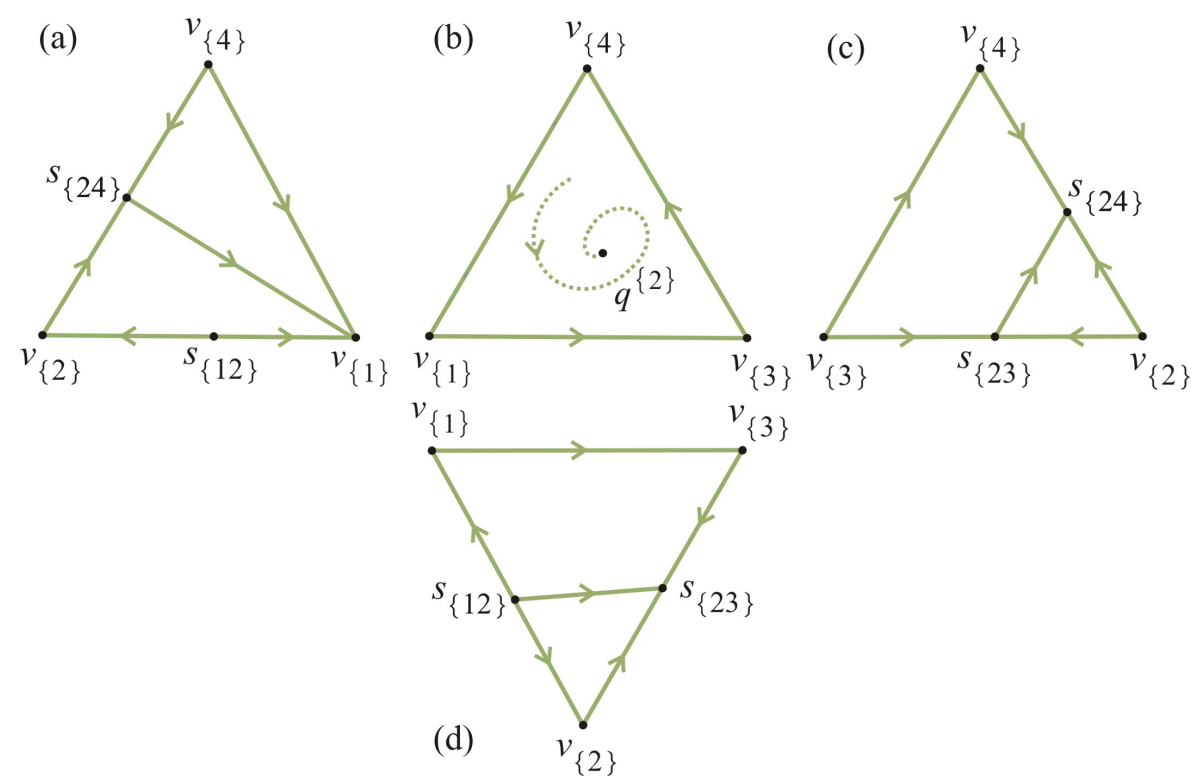

$v_{\{2\}}$

Figure 5: The phase portrait on $\partial \Sigma$ for the Leslie-Gower map $T$ given by 6). (a) The phase portrait on $(\partial \Sigma)_{3}$. (b) The phase portrait on $(\partial \Sigma)_{2}$. (c) The phase portrait on $(\partial \Sigma)_{1}$. (d) The phase portrait on $(\partial \Sigma)_{4}$.

\subsection{Boundary dynamics}

Recall that each coordinate plane $\pi_{i}$ is positively invariant under $T$, and $\left.T\right|_{\pi_{i}}$ is a $3 \mathrm{D}$ Leslie-Gower model which admits a $2 \mathrm{D}$ carrying simplex. Moreover, $\partial \Sigma$ is composed of the 2 D carrying simplices of $\left.T\right|_{\pi_{i}}, i=1,2,3,4$. 
By Lemmas 47 it is easy to check that $\left.T\right|_{\pi_{4}}$ is in class 4 (see Fig. $5(\mathrm{~d})$ ), $\left.T\right|_{\pi_{3}}$ is in class 8 (see Fig. 5 (a)), $\left.T\right|_{\pi_{2}}$ is in class 27 (see Fig. $5(\mathrm{~b})$ ), and $\left.T\right|_{\pi_{1}}$ is in class 9 (see Fig. 5(c)) for all $\mu>0$. It then follows that $s_{\{23\}}$ is globally attracting on the interior of $\pi_{4}$ for $\left.T\right|_{\pi_{4}}, v_{\{1\}}$ is globally attracting on the interior of $\pi_{3}$ for $\left.T\right|_{\pi_{3}}$, and $s_{\{24\}}$ is globally attracting on the interior of $\pi_{1}$ for $\left.T\right|_{\pi_{1}}$. Therefore, the global dynamics is known for $\left.T\right|_{\pi_{4}},\left.T\right|_{\pi_{3}}$ and $\left.T\right|_{\pi_{1}}$, and every nontrivial orbit converges to some boundary fixed point, which is independent of $\mu$. Since $\left.T\right|_{\pi_{2}}$ is in class 27 , the three edges of $(\partial \Sigma)_{2}$ form a heteroclinic cycle and there is a fixed point (i.e. $\left.q^{\{2\}}\right)$ in the interior of $(\partial \Sigma)_{2}$; see Fig. 5(b). The heteroclinic cycle is repelling for $\left.T\right|_{\pi_{2}}$ by Lemma 8 . The Jacobian matrix $\left.D T\right|_{\pi_{2}}\left(q^{\{2\}}\right)$ has eigenvalues $0.8562,0.9901 \pm 0.0395 \mathrm{i}$, which are the three internal eigenvalues of $D T\left(q^{\{2\}}\right)$. Thus, $q^{\{2\}}$ is stable for $\left.T\right|_{\pi_{2}}$. Note that $\left.T\right|_{\pi_{2}}$ is independent of $\mu$, so the boundary dynamics of $T$ is independent of $\mu$, and the dynamics on $\partial \Sigma$ for $T$ is shown in Fig. 5. On the other hand, the external eigenvalue of $D T\left(q^{\{2\}}\right)$ is $\frac{1+\mu}{1+0.9013 \mu}>1$, so $q^{\{2\}}$ is unstable for $T$ when $\mu>0$. Therefore, we conclude that the dynamics on the boundary of the carrying simplex which is shown in Fig. 5 and the stability of the fixed points on the boundary of the carrying simplex do not change as the parameter $\mu$ varies.

\subsection{Evolution of the interior attractor}

In this subsection, we study the evolution of the attractor within $\dot{\Sigma}$ for increasing $\mu$ from zero. We find that chaotic attractors can occur in $\dot{\Sigma}$, and the route to chaos was detected as the parameter $\mu$ is increased. Specifically, in this model

- cascades of quasiperiod-doubling bifurcations can occur which lead to chaos eventually.

Quasiperiod-doubling bifurcation in our context is referred to the phenomenon that a quasiperiodic curve rounding twice bifurcates from the original one. We call the bifurcated quasiperiodic curve a 2-quasiperiodic curve. The similar phenomena have also been observed in [39, 40].

At $\mu \approx 0.1537$, the positive fixed point undergoes a supercritical Neimark-Sacker bifurcation with first Lyapunov coefficient $L_{1}(0)=-0.6667<0$ (see [41, 42]). The fixed point becomes unstable and a stable invariant cycle can occur for $\mu>0.1537$. Fig. 6(a) shows the attracting quasiperiodic curve when $\mu=0.2$. As $\mu$ is increased from 0.2 


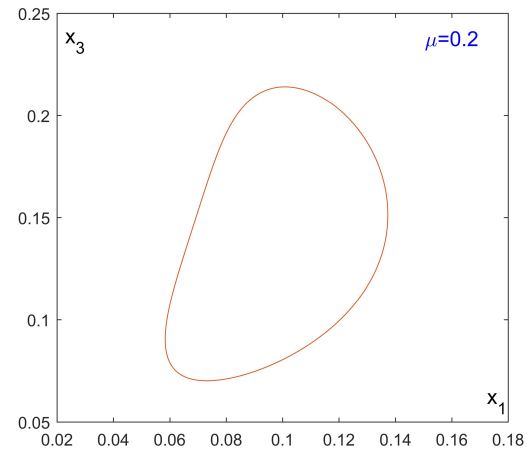

(a)

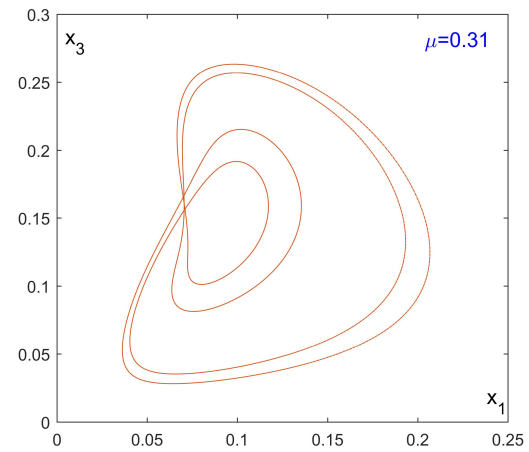

(c)

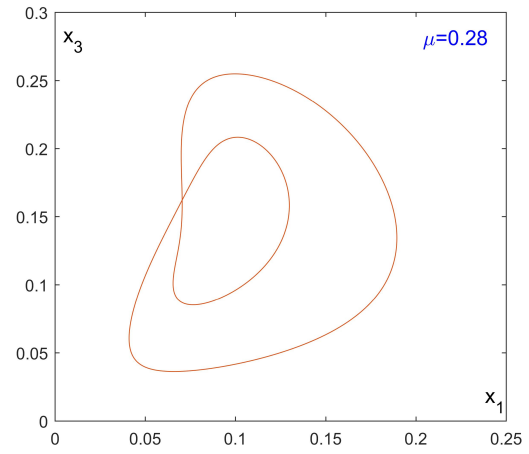

(b)

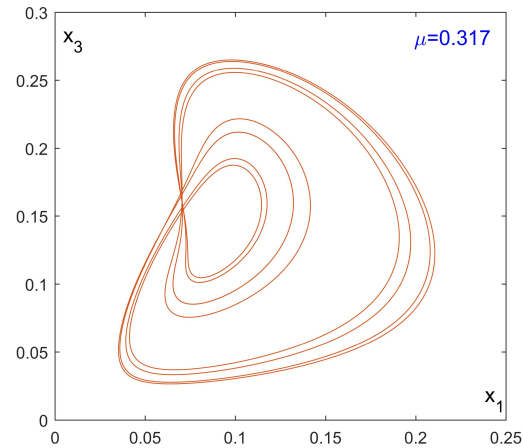

(d)

Figure 6: Evolution of the interior attractor as $\mu$ is increased from 0.2 to 0.317 . (a) The attractor at $\mu=0.2$ is a quasiperiodic curve. (b) The attractor at $\mu=0.28$ is a 2-quasiperiodic curve. (c) The attractor at $\mu=0.31$ is a 4 -quasiperiodic curve. (d) The attractor at $\mu=0.317$ is a 8 -quasiperiodic curve.

the quasiperiodic curve increases in size, until about $\mu=0.246$, where a quasiperioddoubling cascade begins; Figs. 6(b) 6(d) show the attracting 2-quasiperiodic curve, 4-quasiperiodic curve and 8-quasiperiodic curve at $\mu=0.28, \mu=0.31$ and $\mu=0.317$, respectively. Such quasiperiod-doubling cascades eventually lead to chaos at about $\mu=$ 0.3185. Figs. 7(a) 7(f) show the different chaotic attractors for different values of the parameter $\mu>0.3185$.

The corresponding Lyapunov exponents as functions of $\mu$ are shown in Fig. 8 , from which we can see that the largest Lyapunov exponent is positive for some $0.318<\mu<$ 0.494, such as the values of $\mu$ in Fig. 7, which indicates the presence of chaotic attractors. Note that as $\mu$ is increased from 0.318 , there also exist some regions such that the largest 


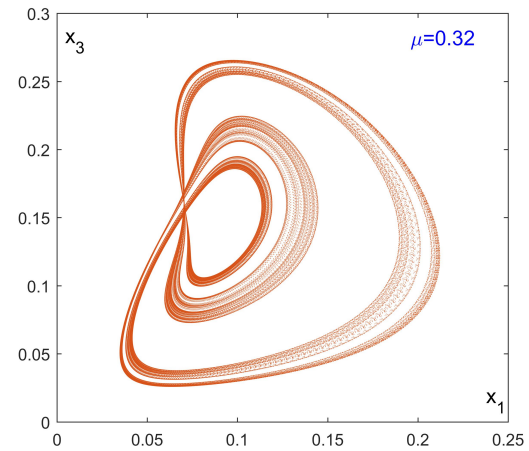

(a)

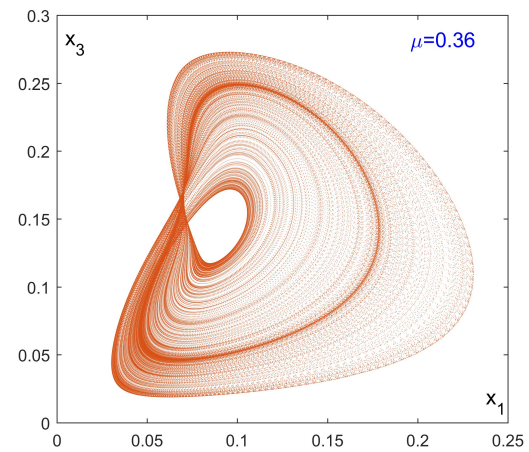

(c)

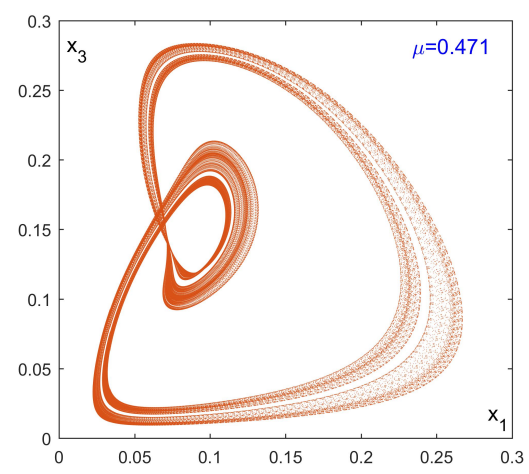

(e)

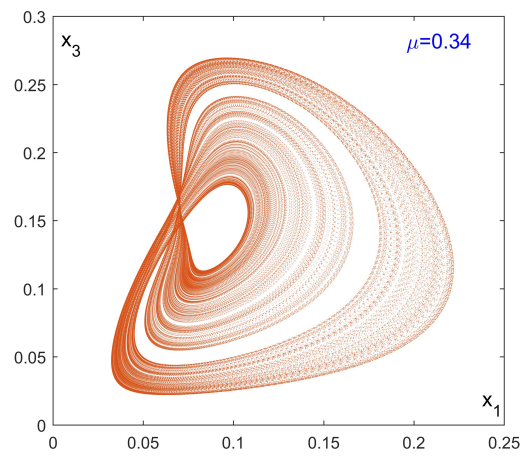

(b)

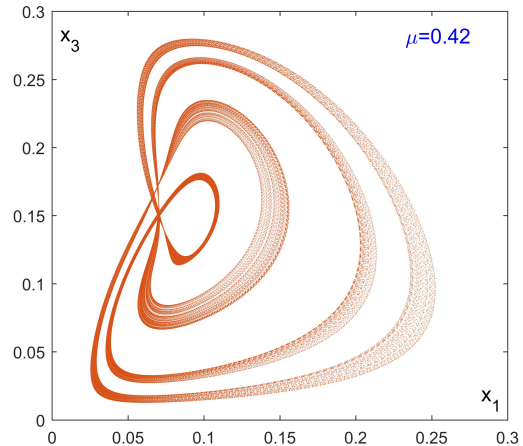

(d)

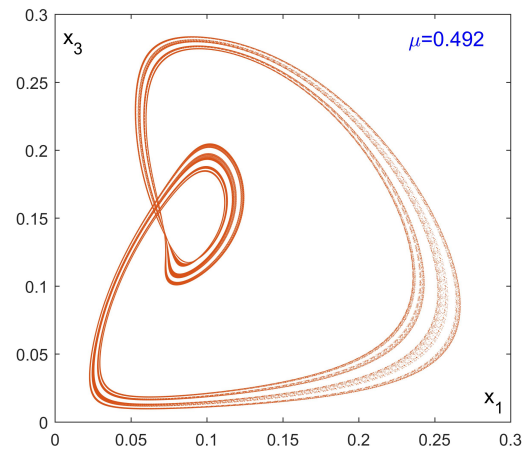

(f)

Figure 7: Evolution of the interior attractor as $\mu$ is increased from 0.32 to 0.492 . (a) The chaotic attractor for $\mu=0.32$. (b) The chaotic attractor for $\mu=0.34$. (c) The chaotic attractor for $\mu=0.36$. (d) The chaotic attractor for $\mu=0.42$. (e) The chaotic attractor for $\mu=0.471$. (f) The chaotic attractor for $\mu=0.492$. The successive iterates $T^{k}$ of $T$ have been applied to the initial point $x^{[0]}=$ $(0.1046,0.01,0.1946,0.0746)^{\tau}$ for each $\mu$, producing a sequence asymptotic to the chaotic attractor. Here, 200000 points of this sequence are plotted, ignoring the first 50000 iterates. 


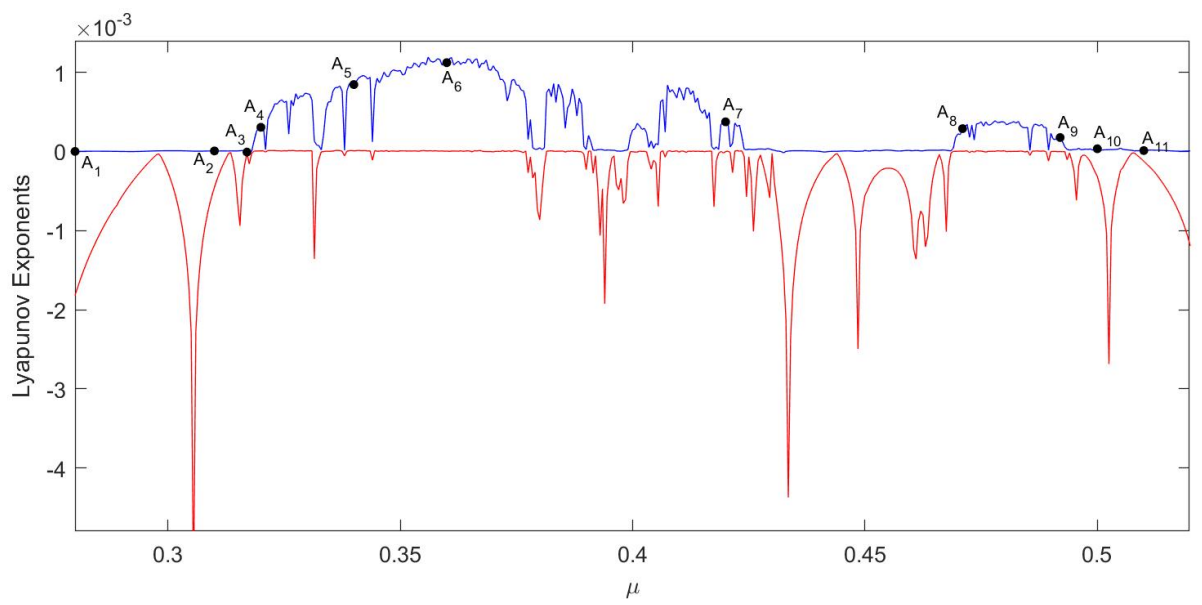

Figure 8: The corresponding largest (blue curve) and the second largest (red curve) Lyapunov exponents as functions of $\mu \in[0.28,0.52]$. The parameter values $\mu$ used in Figs 6 7 with the corresponding largest Lyapunov exponents are marked with the closed dots $\bullet$, where $A_{1}=(0.28,0), A_{2}=(0.31,0)$, $A_{3}=(0.317,0), A_{4}=(0.32,0.0003), A_{5}=(0.34,0.0008), A_{6}=(0.36,0.0011), A_{7}=(0.42,0.0004)$, $A_{8}=(0.471,0.0003), A_{9}=(0.492,0.0002), A_{10}=(0.5,0)$, and $A_{11}=(0.51,0)$. The largest Lyapunov exponents are 0 for $\mu=0.2,0.8,1$, which are not plotted in the figure.

Lyapunov exponent is zero, which are the quasiperiodic windows, i.e., quasiperiodic curves appear again for these values of $\mu$. However, when $\mu>0.494$ the chaotic attractor disappears and an inverse quasiperiod-doubling cascade begins; see Figs. 9(a) 9(d)

Remark 4.1. One can also let another parameter change to observe the evolution of the interior attractor once a chaotic attractor is found. For example, by setting $\mu=0.36$ and fixing all $r_{i}$ and $a_{i j}$ given in (7) and (8) except $r_{1}$, one can find the similar bifurcation scenarios and the evolution of the interior attractor when $r_{1}$ is increased from 0 to 0.4.

\subsection{Invasion can lead to chaos}

Consider the subsystem $\left.T\right|_{\pi_{2}}$ of species 1,3 and 4 given by

$$
\left\{\begin{array}{l}
T_{1}(x)=\frac{\left(1+r_{1}\right) x_{1}}{1+r_{1}\left(a_{11} x_{1}+a_{13} x_{3}+a_{14} x_{4}\right)} \\
T_{3}(x)=\frac{\left(1+r_{3}\right) x_{3}}{1+r_{3}\left(a_{31} x_{1}+a_{33} x_{3}+a_{34} x_{4}\right)} \\
T_{4}(x)=\frac{\left(1+r_{4}\right) x_{4}}{1+r_{4}\left(a_{41} x_{1}+a_{43} x_{3}+a_{44} x_{4}\right)}
\end{array}\right.
$$




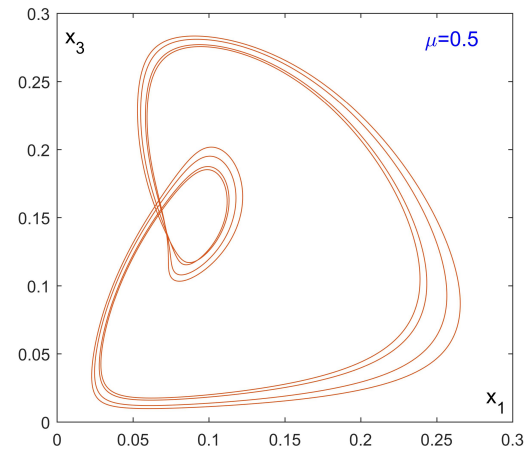

(a)

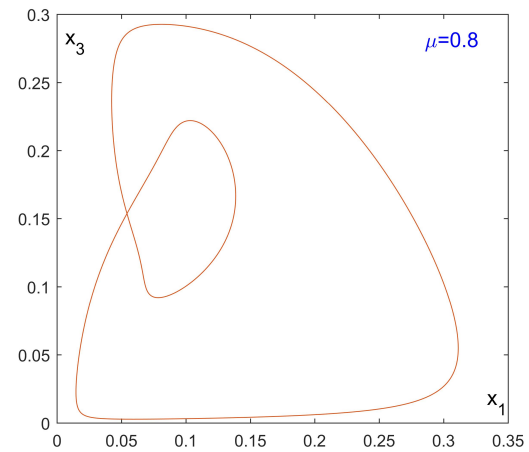

(c)

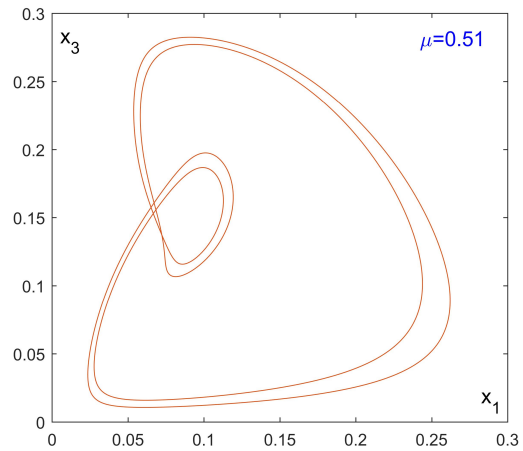

(b)

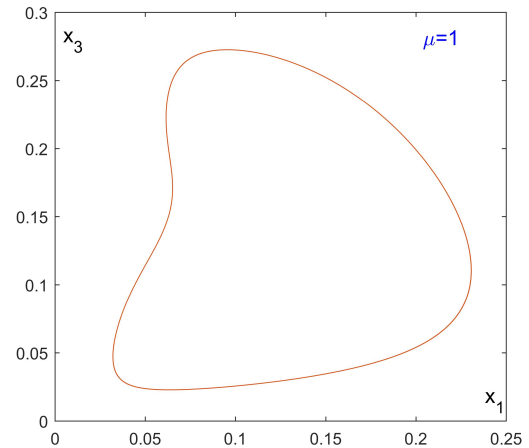

(d)

Figure 9: Evolution of the interior attractor as $\mu$ is increased from 0.5 to 1 . (a) The attractor at $\mu=0.5$ is a 8-quasiperiodic curve. (b) The attractor at $\mu=0.51$ is a 4 -quasiperiodic curve. (c) The attractor at $\mu=0.8$ is a 2 -quasiperiodic curve. (d) The attractor at $\mu=1$ is a quasiperiodic curve.

with $r_{i}, a_{i j}$ given in (7) and (8), $i, j=1,3,4$. Recall that $\left.T\right|_{\pi_{2}}$ is in the class 27, so the axial fixed point $v_{\{1\}}$ is stable in the $x_{1} x_{4}$ subspace and unstable in the $x_{1} x_{3}$ subspace, the axial fixed point $v_{\{3\}}$ is stable in the $x_{1} x_{3}$ subspace and unstable in the $x_{3} x_{4}$ subspace, and the axial fixed point $v_{\{4\}}$ is stable in the $x_{3} x_{4}$ subspace and unstable in the $x_{1} x_{4}$ subspace. In particular, the boundary of $(\partial \Sigma)_{2}$ is a heteroclinic cycle (see Fig. 5(b)), which is due to the competition relations

$$
\begin{aligned}
& a_{11}>a_{31}, a_{13}>a_{33}, \\
& a_{33}>a_{43}, a_{34}>a_{44}, \\
& a_{41}>a_{11}, a_{44}>a_{14} .
\end{aligned}
$$

In adaptive dynamics, the relations in (14) mean that the species 3 can invade species 1 (in the absence of species 4) but not vice versa, and the outcome is that species 1 is 
driven to extinction, whilst species 3 remains extant; the species 4 can invade species 3 (in the absence of species 1) but not vice versa, and the outcome is that species 3 is driven to extinction, whilst species 4 remains extant; the species 1 can invade species 4 (in the absence of species 3) but not vice versa, and the outcome is that species 4 is driven to extinction, whilst species 1 remains extant; see Remark 3.1. This means that the species 1 , 3 and 4 compete in the rock-paper-scissors manner under the Leslie-Gower dynamics described by $\left.T\right|_{\pi_{2}}$. However, there is a stable fixed point $q^{\{2\}} \approx(0.1046,0,0.1946,0.0746)^{\tau}$ for $\left.T\right|_{\pi_{2}}$, so the species 1,3 and 4 can coexist at the stable steady state $q^{\{2\}}$.

The outcomes of invasion attempts by an invader (i.e. species 2) into the trimorphic resident population set by species 1,3 and 4 at the steady state $q^{\{2\}}$ are of particular interest. To begin with, the trimorphic resident population is at the steady state $q^{\{2\}}$ and then the invader species 2 is introduced in small quantities. Since the external eigenvalue of $D T\left(q^{\{2\}}\right)$ is $\frac{1+\mu}{1+0.9013 \mu}>1$, which implies the invasion fitness of species 2 in an environment set by species 1,3 and 4 at the steady state $q^{\{2\}}$ given by $\ln \frac{1+\mu}{1+0.9013 \mu}$ is positive for all $\mu>0$, the species 2 can always successfully invade such a trimorphic resident population. Moreover, since $\left.T\right|_{\pi_{2}}$ is independent of $\mu$, the environment set by the resident species 1,3 and 4 at the steady state $q^{\{2\}}$ is independent of $\mu$. This allows one to study the possible outcomes of invasion attempts by the invader species 2 into such a fixed environment for different values of $\mu$.

Our results in section 4.2 show that the outcomes of the invasion attempts by the species 2 will be different as the change of the parameter $\mu$. For example, for $\mu<0.1537$, the Leslie-Gower model $T$ given by (6) of the four competing species has a stable positive fixed point $p$ which means a coexistence steady state of the invader species 2 and the resident species 1, 3 and 4 ; for $\mu=0.2$, the model $T$ has an attracting quasiperiodic curve, which means a quasiperiodic coexistence of the invader species 2 and the resident species 1, 3 and 4 ; for $\mu=0.36$, the model $T$ has a chaotic attractor which means a chaotic coexistence of the invader species 2 and the resident species 1, 3 and 4 . Importantly, our results show that the invasion by an invader into a trimorphic population under the Leslie-Gower dynamics can lead to chaos. 


\section{Discussion}

This paper presents a case study of chaos in the classical Leslie-Gower model of four competing species induced by the map (6). The selection of parameters is based on a brute-force numerical search in the parameter space consisting of $r_{i}$ and $a_{i j}$ such that the largest Lyapunov exponent of the orbit with the given initial value is positive. The numerical result shows that the largest Lyapunov exponent is positive when $r_{i}$ and $a_{i j}$ are equal to the values in (7) and (8) with $\mu=0.32$, which implies the occurrence of chaos. We then explore the mechanism of occurrence of chaos in the 4D Leslie-Gower map, especially the route to chaos. To begin with, we choose the parameters $r_{i}, a_{i j}$ given in (7) and (8) with $\mu=0.32$ such that the $4 \mathrm{D}$ Leslie-Gower map has a chaotic attractor. We notice that species 1, 3 and 4 competing in the rock-paper-scissors manner can coexist in a stable steady state $q^{\{2\}}$ which is independent of the parameter $\mu$, so we then fix all $r_{i}, a_{i j}$ in (7) and (8) except $\mu$. By selecting such parameters, in addition to observing the evolution of the interior attractor, one can observe the possible outcomes of invasion attempts by the invader species 2 into a fixed environment set by the resident species 1 , 3 and 4 at the steady state $q^{\{2\}}$ as $\mu$ varies.

A quasiperiod-doubling route to chaos is found in the $4 \mathrm{D}$ Leslie-Gower map (6) as $\mu$ is increased from 0 to 1 . Specifically, increasing $\mu$ from 0 , the map undergoes a supercritical Neimark-Sacker bifurcation, which bifurcates a stable quasiperiodic curve, and then the map undergoes a cascade of quasiperiod-doubling bifurcations, and after a critical point becomes chaotic, as illustrated in Fig. 6 and Fig. 7. Biologically, the results indicate a possible outcome of invasion attempts by an invader (species 2) into the environment set by the resident species 1,3 and 4 at a steady state which compete in the rock-paperscissors manner under the Leslie-Gower dynamics, that is the invasion can lead to chaos. However, the outcomes of the invasion might be different as the parameter $\mu$ varies, that is the invasion may lead to quasiperiodic behavior or chaotic behavior which depends on the value of the parameter $\mu$; see Figs. 69.

Since the 4D Leslie-Gower map has a 3D carrying simplex $\Sigma$, the chaotic attractor is contained in the interior of this invariant manifold. This means that the 4D Leslie-Gower map can admit a 3D carrying simplex containing a chaotic attractor. In order to have a clearer understanding of the dynamics on the carrying simplex, we further study the 
dynamics on the boundary of the carrying simplex in detail. The study shows that the boundary dynamics which is independent of the parameter $\mu$ is determined by the classes 4, 8, 9 and 27 provided by Jiang and Niu in [9] for the 3D Leslie-Gower map. The phase portrait on the boundary of the carrying simplex is shown in Fig. 5. Therefore, in the $4 \mathrm{D}$ Leslie-Gower map (6), as $\mu$ is increased from 0 , the interior attractor evolves from a fixed point to a quasiperiodic curve, and then from a quasiperiodic curve to a chaotic attractor, but the boundary dynamics remains unchanged.

Recall that the carrying simplex $\Sigma$ carries all the asymptotic dynamics, and the 4D Leslie-Gower competitive map restricted to $\Sigma$ is a 3D homeomorphism, so the 4D LeslieGower map actually behaves like a 3D homeomorphism. On the other hand, for the 1D and 2D Leslie-Gower maps, every orbit converges to a fixed point, so there is no chaos. The 3D Leslie-Gower map which behaves like a 2D homeomorphism was classified into 33 stable equivalence classes via the boundary dynamics on the carrying simplex in 9 , and it was proved that every orbit converges to a fixed point and hence chaos cannot occur in classes $1-25$ and 33 ([9, 13, 15]). In classes $26-31$, Neimark-Sacker bifurcations can occur which cannot occur in class 32. However, the 3D Leslie-Gower map (2) presents the similar dynamic scenarios as the 3D Lotka-Volterra competition model (5) [18, 19] in which chaos cannot occur due to the carrying simplex theory [1, 43] and the PoincaréBendixson theorem. Moreover, we have carried out a brute-force numerical search in the 3D Leslie-Gower map but no chaotic attractor has been observed. Therefore, by the numerical experiment and the comparison between the 3D Leslie-Gower map (2) and the 3D Lotka-Volterra competition model (5), we conjecture that chaos may not occur in the 3D Leslie-Gower map and the 4D Leslie-Gower map is probably the simplest example that might have chaos.

Note that the Poincaré-Bendixson theorem does not hold for discrete-time dynamical systems. To the best of our knowledge, the available results on nonexistence of chaos for 3D competitive maps with a carrying simplex are the following: Ruiz-Herrera 6] proved that every orbit converges to a fixed point when there is no positive fixed point; Niu and Ruiz-Herrera [13] proved that every orbit converges to a fixed point when there is a unique positive fixed point whose index is -1 ; Balreira, Elaydi and Luís [14] and Gyllenberg, Jiang and Niu 15 provided criteria on the global stability of the unique 
positive fixed point under special conditions. However, it should be pointed out that how to prove there is no chaos in the 3D Leslie-Gower competitive map is still a very difficult problem, which is left for future research.

\section{Acknowledgments}

The authors are greatly indebted to three referees for the careful and patient reading of our original manuscript, many valuable comments and useful suggestions which led to much improvement in the presentation of our results.

This work is supported by the National Natural Science Foundation of China (NSFC) under Grant No. 11371252 and Grant No. 11771295, Shanghai Gaofeng Project for University Academic Program Development, and the Academy of Finland.

\section{References}

[1] M. W. Hirsch, Systems of differential equations which are competitive or cooperative. III: Competing species, Nonlinearity 1 (1988) 51-71.

[2] H. L. Smith, Periodic competitive differential equations and the discrete dynamics of competitive maps, J. Differential Equations 64 (1986) 165-194.

[3] Y. Wang, J. Jiang, Uniqueness and attractivity of the carrying simplex for discrete-time competitive dynamical systems, J. Differential Equations 186 (2002) 611-632.

[4] O. Diekmann, Y. Wang, P. Yan, Carrying simplices in discrete competitive systems and agestructured semelparous populations, Discrete Contin. Dyn. Syst. 20 (2008) 37-52.

[5] M. W. Hirsch, On existence and uniqueness of the carrying simplex for competitive dynamical systems, J. Biol. Dyn. 2 (2008) 169-179.

[6] A. Ruiz-Herrera, Exclusion and dominance in discrete population models via the carrying simplex, J. Difference Equ. Appl. 19 (2013) 96-113.

[7] S. Baigent, Convexity of the carrying simplex for discrete-time planar competitive Kolmogorov systems, J. Difference Equ. Appl. 22 (2016) 609-622.

[8] J. Jiang, L. Niu, Y. Wang, On heteroclinic cycles of competitive maps via carrying simplices, J. Math. Biol. 72 (2016) 939-972.

[9] J. Jiang, L. Niu, On the equivalent classification of three-dimensional competitive Leslie/Gower models via the boundary dynamics on the carrying simplex, J. Math. Biol. 74 (2017) 1223-1261.

[10] M. Gyllenberg, J. Jiang, L. Niu, P. Yan, On the classification of generalized competitive AtkinsonAllen models via the dynamics on the boundary of the carrying simplex, Discrete Contin. Dyn. Syst. 38 (2018) 615-650. 
[11] M. Gyllenberg, J. Jiang, L. Niu, P. Yan, Permanence and universal classification for discrete-time competitive systems via the carrying simplex, submitted.

[12] J. Jiang, L. Niu, On the equivalent classification of three-dimensional competitive Atkinson/Allen models relative to the boundary fixed points, Discrete Contin. Dyn. Syst. 36 (2016) 217-244.

[13] L. Niu, A. Ruiz-Herrera, Trivial dynamics in discrete-time systems: carrying simplex and translation arcs, Nonlinearity 31 (2018) 2633-2650.

[14] E. C. Balreira, S. Elaydi, R. Luís, Global stability of higher dimensional monotone maps, J. Difference Equ. Appl. 23 (2017) 2037-2071.

[15] M. Gyllenberg, J. Jiang, L. Niu, A note on global stability of three-dimensional Ricker models, J. Difference Equ. Appl. 25 (2019) 142-150.

[16] P. Liu, S. Elaydi, Discrete competitive and cooperative models of Lotka-Volterra type, J. Comp. Anal. Appl 3 (2001) 53-73.

[17] J. M. Cushing, S. Levarge, N. Chitnis, S. M. Henson, Some discrete competition models and the competitive exclusion principle, J. Difference Equ. Appl. 10 (2004) 1139-1151.

[18] M. L. Zeeman, Hopf bifurcations in competitive three-dimensional Lotka-Volterra systems, Dynam. Stability Systems 8 (1993) 189-216.

[19] P. van den Driessche, M. L. Zeeman, Three-dimensional competitive Lotka-Volterra systems with no periodic orbits, SIAM J. Appl. Math. 58 (1998) 227-234.

[20] D. Xiao, W. Li, Limit cycles for the competitive three dimensional Lotka-Volterra system, J. Differential Equations 164 (2000) 1-15.

[21] E. C. Zeeman, M. L. Zeeman, From local to global behavior in competitive Lotka-Volterra systems, Trans. Amer. Math. Soc. 355 (2002) 713-734.

[22] Z. Lu, Y. Luo, Three limit cycles for a three-dimensional Lotka-Volterra competitive system with a heteroclinic cycle, Comp. Math. Appl. 46 (2003) 231-238.

[23] M. Gyllenberg, P. Yan, Y. Wang, A 3D competitive Lotka-Volterra system with three limit cycles: A falsification of a conjecture by Hofbauer and So, Appl. Math. Lett. 19 (2006) 1-7.

[24] Z. Hou, S. Baigent, Fixed point global attractors and repellors in competitive Lotka-Volterra systems, Dynamical Systems 26 (2011) 367-390.

[25] S. Baigent, Z. Hou, Global stability of interior and boundary fixed points for Lotka-Volterra systems, Diff. Eq. Dyn. Syst. 20 (2012) 53-66.

[26] S. Baigent, Geometry of carrying simplices of 3-species competitive Lotka-Volterra systems, Nonlinearity 26 (2013) 1001-1029.

[27] X. Chen, J. Jiang, L. Niu, On Lotka-Volterra equations with identical minimal intrinsic growth rate, SIAM J. Applied Dyn. Sys. 14 (2015) 1558-1599.

[28] J. Jiang, L. Niu, On the validity of Zeeman's classification for three dimensional competitive differential equations with linearly determined nullclines, J. Differential Equations 263 (2017) 7753-7781.

[29] N. Neirynck, B. Al-Hdaibat, W. Govaerts, Y. A. Kuznetsov, H. G. E. Meijer, Using MatContM in the study of a nonlinear map in economics, J. Phys.: Conf. Ser. 692 (2016) 012013.

[30] A. Dhooge, W. Govaerts, Y. A. Kuznetsov, H. G. E. Meijer, B. Sautois, New features of the software 
MatCont for bifurcation analysis of dynamical systems, MCMDS 14 (2008) 147-175.

[31] J.-P. Eckmann, D. Ruelle, Ergodic theory of chaos and strange attractors, Rev. Mod. Phys. 57 (1985) 617-656.

[32] H. F. V. Bremen, F. E. Udwadia, W. Proskurowski, An efficient QR based method for the computation of Lyapunov exponents, Phys. D 101 (1997) 1-16.

[33] P. Frederickson, J. L. Kaplan, E. D. Yorke, J. A. Yorke, The Liapunov dimension of strange attractors, J. Differential Equations 49 (1983) 185-207.

[34] S. A. H. Geritz, Resident-invader dynamics and the coexistence of similar strategies, J. Math. Biol. 50 (2005) 67-82.

[35] S. A. H. Geritz, M. Gyllenberg, F. J. A. Jacobs, K. Parvinen, Invasion dynamics and attractor inheritance, J. Math. Biol. 44 (2002) 548-560.

[36] S. A. H. Geritz, E. Kisdi, G. Meszéna, J. A. J. Metz, Evolutionarily singular strategies and the adaptive growth and branching of the evolutionary tree, Evolutionary Ecology 12 (1998) 35-57.

[37] S. A. H. Geritz, J. A. J. Metz, E. Kisdi, G. Meszéna, Dynamics of adaptation and evolutionary branching, Phys. Rev. Letters 78 (1997) 2024-2027.

[38] J. Mierczyński, L. Niu, A. Ruiz-Herrera, Linearization and invariant manifolds on the carrying simplex for competitive maps, J. Differential Equations (2019) in press.

[39] L. Gardini, R. Lupini, C. Mammana, M. G. Messia, Bifurcations and transitions to chaos in the three-dimensional Lotka-Volterra map, SIAM J. Appl. Math. 47 (1987) 455-482.

[40] L. van Veen, The quasi-periodic doubling cascade in the transition to weak turbulence, Phys. D 210 (2005) 249-261.

[41] Y. A. Kuznetsov, R. J. Sacker, Neimark-Sacker bifurcation, Scholarpedia 3 (2008) 1845.

[42] W. Govaerts, Y. A. Kuznetsov, H. G. E. Meijer, N. Neirynck, A study of resonance tongues near a Chenciner bifurcation using MatcontM, in: European Nonlinear Dynamics Conference, 2011, pp. $24-29$.

[43] H. L. Smith, Monotone Dynamical Systems: An Introduction to the Theory of Competitive and Cooperative Systems, in: Math. Surveys Monogr., Vol. 41, Amer. Math. Soc., Providence, RI, 1995. 